\title{
DEVELOPMENT AND PSYCHOMETRIC PROPERTIES OF PARENTAL EXPRESSED EMOTIONS SCALE
}

\author{
Shammem Akhtar, Zaqia Bano \\ University of Gujrat, Gujrat Pakistan
}

\begin{abstract}
Objective: To construct a scale and psychometric properties for the assessment of Parental expressed emotions scale in Urdu language.

Study Design: Cross sectional study.

Place and Duration of Study: Department of Psychology, University of Gujrat, from Mar to Oct 2019.

Methodology: The initial item pool of 224 items was generated with the help of CFI (Camber well Family Interview description of parental expressed emotions scale, literature review and three focus groups with target population. Among the 124 expert evaluated items after pilot study 100 items were retained which included the five dimensions of parental expressed emotions scale including, Criticalcomments, Hostility, Emotional over involvement, Warmth and Positive remarks. Furthermore, in the final administration of this scales data were collected from 380 parents (both mothers \& fathers) from the educational institutions and community of Gujrat using self-reported questionnaire. The exploratory factor analysis (EFA), confirmatory factor analysis (CFA) and reliability analysis were implied for data scrutiny.

Results: The final administration of 100 items was handed over to 380 participants. The model fit showed a $p$-value of 0.000 that established the structure validity and significance of the items to its subscales. At the final stage among the 100 items 31 were reliable for further use.

Conclusion: A scale to measure parental expressed emotions in Urdu language is competently established with 31 questions andfive sub-scales.
\end{abstract}

Keywords: Critical comments, Expressed emotions, Hostility, Emotional over involvement, Warmth, Positive remarks.

This is an Open Access article distributed under the terms of the Creative Commons Attribution License (http://creativecommons.org/licenses/by/4.0), which permits unrestricted use, distribution, and reproduction in any medium, provided the original work is properly cited.

\section{INTRODUCTION}

In daily lives emotions play a very crucial role and human characteristics of expressing emotions are similar despite of differences in culture, language and ecology ${ }^{1}$. A number of different ways are used to express emotions, including verbal as well as non-verbal although facial expressions are one of the most important way that express emotions ${ }^{2}$.

Expressed emotion is a term used in mental health to denote the intensity of expression of a range of emotions within the family context. Levels of expressed emotion may be high or low. This emotion may be considered 'negative or high' (for example hostility, critical comments \& Emotional over involvement) and positive or low

Correspondence: Dr Shammem Akhtar, PhD Scholar, Psychology Department, University of Gujrat, Gujrat Pakistan

Received: 02 Dec 2019; revised received: 25 Apr 2020; accepted: 03 May 2020 expressed emotions (warmth and positive regards). In a family parents play very important role in the children growth as well as in personality development, in home environment parents can be assessed with high expressed emotions, and with low expressed emotions. To understand the origins of the concept "Expressed Emotion," one has to go back to the 1950s for the seminal works by George Brown who studied the relapse of schizophrenia. However, many of these patients were to be readmitted soon after discharge due to symptom relapse. To understand the basis for the symptom relapse they take interview from the family and find family expressed emotions are cause of symptoms relapse ${ }^{3}$.

Parental Expressed emotions can be defined as hostility, emotional over-involvement, attitudes of high criticism, warmth, and positive regards of the care givers ${ }^{4}$. Mostly parents 
expressed their emotions through critical comments and can be assessed as an increased tone, volume, tempo that cause frustration. intentionally it become problems for others members in home and the caregivers fail to recognize that their attitude could be potential indicators of psychotic disorder in children. This shown in the fact that $70 \%$ of critical comments were found to be cause of negative symptoms of schizophrenia relatively than hallucinations and delusions ${ }^{5}$.

Hostility is the second one element in parental expressed emotions, Caregivers state that client is a cause of problems, and they show act of shouting and anger. Critical comments rated as being present or absent during the assessment. Consequence of anger and annoyance followed via critical comments and denial of the client. Family attitude of general criticism and rejections attitude rated as hostility ${ }^{6}$.

Third element of expressed emotions is the emotional over involvement. It reveals by excessive self-sacrifice over-identification, emotionally over involved and extreme over-protective conduct with the client. Caregivers take responsibility for everything, they think that all is their mistake, show pity, they don't want the client perform his own daily activities. They show selfignoring behavior and give less importance to themselves ${ }^{7}$.

Further, warmth measured on the basis of kindness, caregiver expressed empathy and concern regarding client. It depends greatly on verbal qualities with pleased and common complement, which mostly express an empathic attitude by the care givers. While Positive regard consist of statements that shows support and appreciation or for client's behavior and verbal as well as nonverbal support by the close relative. The statement by the care givers that they feel very near to the client, they appreciate client 's slight initiation or efforts in his daily activities, they state that they love being with him/her ${ }^{8}$.

Previously parental expressed emotions scales have been used for relapse of psychiatric disorders. Some others measuring tools also used to assess expressed emotions as the five-minute speech sample is similar to the camber well family interview but warmth and hostility are not assessed in this interview ${ }^{9}$.

In the same ways Family attitude scale selfreport measure of expressed emotions which emphasizes only on the criticism and hostility ${ }^{10}$.

The camber well family interview, only one model that gives all five elements of expressed emotions those are Criticism, Hostility, EOI, Warmth, and Positive Remarks ${ }^{11}$.

These indications represented a dire need to develop culturally valid assessment tool to measure parental expressed emotions scale. To overcome these flaws, the present study on the development and psychometric properties of parental expressed emotions scale behavior scale will be a building block. In this situation the present study would be a great breakthrough in the ground of psychological measures as it would construct parental expressed emotions scale in native language. The existing study conducted with an objective to develop an assessment of parental expressed emotions in Urdu language.

\section{METHODOLOGY}

This is a cross sectional analytical studythat was conducted in the department of Psychology, University of Gujrat from Mar 2019 to Oct 2019. The data was collected from various educational institutions and community of Gujrat. The 380 parents (both mothers \& fathers) using convenient sampling. At initial stage an item pool of 224 questions was generated by the investigator with the help, ofcamber well family interview description of parental expressed emotions scale, review of literature and 3 focus group (fig-1). During expert evaluation, 124 items were deleted. Hence, a pilot study was conducted with 100 items. After analysis 31 items were considered as reliable for final administration with five subscales of parental expressed emotions scale, (1) Critical comments, (2) Hostility, (3) Emotional over involvement, (4) Warmth, (5) Positive remarks. 
Current study was approved and permitted by the Ethics Committee of the Department of Psychology, Universityof Gujrat. In pilot study 100 participants were included while final study was conducted on 380 participants. After the permission of institutional authorities' researcher used convenient sample to recruit study partici-

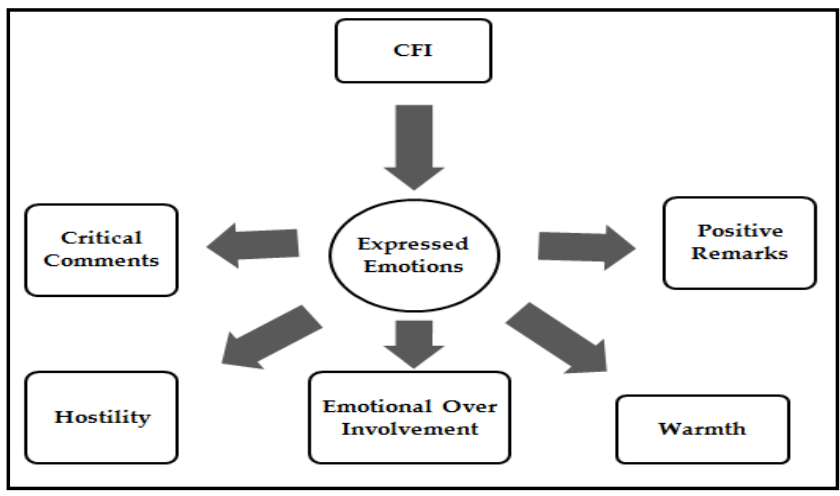

Figure-1: CFI Model (Camberwell Family Interview) Family Expressed Emotions (Leff, J. \& Vaughn 1985) ${ }^{11}$.

pants. Further, the purpose of study was explained to participants and data were collected after taking a written informed consent. The respondents were thoroughly instructed about how torespond to the statements. The respondent's identity confidentiality of data was insured. At the end they were thanked for their participation.

\section{Data Analysis}

Data Analysis to analyze data exploratory factor analysis, confirmatory factor analysis, and reliability analysis were implied by using Statistical Packagefor Social Sciences (SPSS-23) and Analysis of a Moment Structures (AMOS-23) for windows.

\section{RESULTS}

After conducting pilot study 100 items were further administrated on340 parents. From 150 questions 31 were retained after exploratory factor analysis while deleting the irrelevant question. Table-I indicated the KMO is 0.858 which is highly acceptable and Bartlett's Test of Sphericity values is also significant at 0.000 . Table-II depicted number of items in each subscale as item number 7, 8,9,20 and 29 were reflected as reliable for the sub-scale of the warmth (Warmth). Fur- ther, the item number 2,6,8,10,11,13 \& 14 were reliable for emotional over involvement (EMOI). Item number 3,5,6,7,9 \& 10 were reliable for Positive Remarks (POSR). Same way item number 4, $10,18,19,23,25 \& 27$ were reliable for Hostility (HS) Last of all, the item number 23, 24 and \& 42 were reliable for Critical comments (CR). Confir-

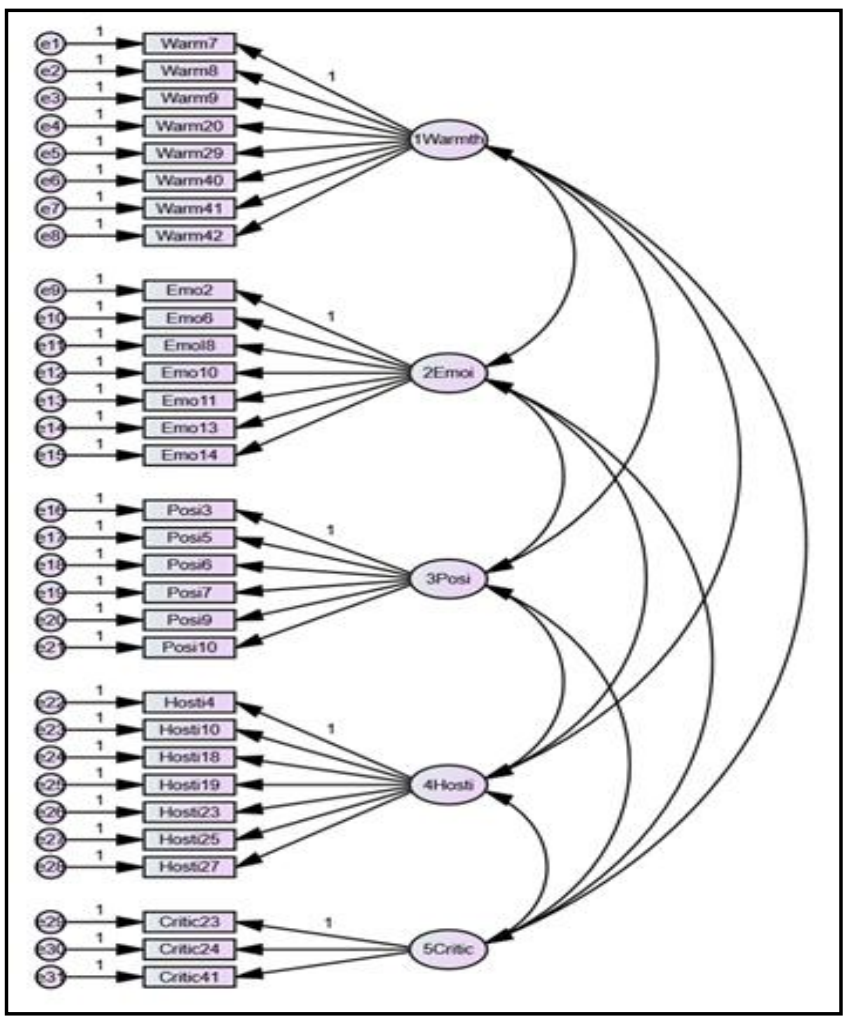

Figure-2: Scale and its subscales.

matory Factor Analysis (CFA) on 31 items appeared significant for five subscales. The value of Comparative Fit Index (CFI) was 0.914 that was in the acceptable range with the $p$-value of 0.000 $<0.05$. The results confirmed the model fit for parental expressed emotions scale.

Reliability of scale was found out by computed Cronbach alpha. Cronbach alpha value was 0.834 . As acceptable reliability value is 0.70 and above. So that in the present study the reliability value is above than acceptable value (table-IV).

\section{DISCUSSION}

The primary objective of current study was to established parental expressed emotions scale in Urdu language and its psychometric proper- 
ties. At initial stage an item pool of 224 questions was generated after expert's evaluation 100 item were deleted. Hereafter, a pilot study was conducted with 124 items in which 100 items were considered as reliable for final administration
Root Mean Square Error of Approximation (RMSEA) is an absolute fit measurement established on the non-centrality factor and is one of the greatest, if it is not the most, reported dimension for model fit ${ }^{15}$.

Table-I: Kaiser-Meyer-Olkin Measure of Sampling Adequacy (KMO) and Bartlett's Test of Sphericity for parental expressed emotions scale $(n=380)$.

\begin{tabular}{|c|c|c|c|c|c|c|c|c|c|}
\hline \multicolumn{4}{|l|}{ Measure } & \multicolumn{2}{|c|}{ KMO } & \multicolumn{4}{|c|}{ Bartlett's Test ofSphericity } \\
\hline \multicolumn{4}{|c|}{ Parental Expressed Emotions Scale } & & & \multicolumn{4}{|c|}{0.000} \\
\hline \multicolumn{10}{|c|}{ Table-II: Factor loading of parental expressed emotions scale $(n=380)$. } \\
\hline Questions & Warmth & Questions & EOI & Questions & POSR & Questions & Hostility & Questions & Critic \\
\hline 7 & 0.637 & 2 & 0.418 & 3 & 0.685 & 4 & 0.402 & 23 & 0.752 \\
\hline 8 & 0.662 & 6 & 0.669 & 5 & 0.773 & 10 & 0.415 & 24 & 0.642 \\
\hline 9 & 0.652 & 8 & 0.685 & 6 & 0.500 & 18 & 0.653 & 42 & 0.563 \\
\hline 20 & 0.576 & 10 & 0.771 & 7 & 0.598 & 19 & 0.640 & & \\
\hline \multirow[t]{3}{*}{29} & 0.600 & 11 & 0.739 & 9 & 0.690 & 23 & 0.678 & & \\
\hline & & 13 & 0.585 & 10 & 0.701 & 25 & 0.733 & & \\
\hline & & 14 & 0.618 & & & 27 & 0.555 & & \\
\hline
\end{tabular}

\begin{tabular}{l|c|c|c|c|c}
\hline \multicolumn{2}{l}{ Table-III: Model Fit Summary of 31 Items (n=380). } \\
\hline$p$-value & $\begin{array}{c}\text { Chi } \\
\text { Square }\end{array}$ & $\begin{array}{c}\text { Goodness of Fit } \\
\text { Index }\end{array}$ & $\begin{array}{c}\text { Comparative Fit } \\
\text { Index }\end{array}$ & $\begin{array}{c}\text { Root Mean Square Error } \\
\text { ofApproximation }\end{array}$ & $\begin{array}{c}\text { Root Mean } \\
\text { Square Residual }\end{array}$ \\
\hline 0.000 & 678.931 & 0.897 & 0.914 & 0.40 & 0.528 \\
\hline
\end{tabular}

Table-IV: Reliabilities of the Subscales $(n=380)$.

Sub Scales

with five sub-scales. Furthermore, the exploratory factor analysis abridged the items to 31 that confirmed by confirmatory factor analysis considered reliable for further use. Current study reported 0.858 KMO value and .000 Bartlett's test of sphericity which indicated the sample size is adequate and data is suitable for further exploratory factor analysis. A study described that appropriateness of data depends on two key factors first is KMO value which determine whether your sample is adequate or not. Second, Bartlett's test of sphericity should be significant which tell us nature of correlation among variables ${ }^{12,13}$.

Moreover, another study explored the KMO value between 0.7 to 0.8 considered good, while value between 0.8 to 0.9 and greater are excellent. In current study the reliability value of final 31 items were 0.858 that was greater than acceptable value. Pervious study is in lined to support the reliability of findings of current study that argued the reliability value of .70 or greater is considered as statistically suitable ${ }^{14}$.

\begin{tabular}{|c|c}
\hline otal Items & Cronbach Alpha r \\
\hline 31 & 0.834
\end{tabular}

Furthermore, confirmatory factor analysis was implied in current study to confirm the factors of study results reported goodness of model fit as values $\mathrm{GFI}=0.897, \mathrm{CFI}=0.914, \mathrm{RMSEA}=0.040$ and $\mathrm{RMSR}=0.0528$, respectively. These results are supported by previous findings stating that acceptable or good RMSEA values should be $>0.05$ Further GFI and CFI values near to 0.9 demonstrate a comparatively good fit 16,17 .

Besides, the established measures were reflected as best fitted and reliable if the value of CFI was $>0.90$. As mentioned earlier, reliability value of 0.70 or greater is considered as statistically significant. The CFI values of parental expressed emotions scale for were 0.914 with the reliability of 0.834 . According to the statistically adequate range the values of newly developed parental expressed emotions scale in highly acceptable ranges.

\section{CONCLUSION}

A scale to gauge parental expressed emotions scale in Urdu language is competently 
established with 31 questions and five sub-scales. It will be very useful for measuring parental expressed emotions.

\section{CONFLICT OF INTEREST}

This study has no conflict of interest to be declared by any author.

\section{REFERENCES}

1. Sauter DA, Eisner F, Ekman P, Scott SK. Cross-cultural recognition of basic emotions through nonverbal emotional vocalizations. Proc Natl Acad Sci U S A 2010; 107(6): 2408-12.

2. Weissman MM, Gammon GD, John K, Merikangas KR, Warner $\mathrm{V}$, Prusoff BA, et al. Children of depressed parents. Increased psychopathology and early onset of major depression. Arch Gen Psychiatry 1987; 44(10): 847-53.

3. Amaresha AC. Expressed emotion in schizophrenia: an overview. Indian J Psychol Med 2012; 34(1): 12-20.

4. Peris TS, Miklowitz DJ. Parental expressed emotion and youth psychopathology: new directions for an old construct. Child Psychiatry Hum Dev 2015; 46(6): 863-73.

5. Vaughn C, Leff J. The measurement of expressed emotion in the families of psychiatric patients. Br J Soc Clin Psychol 1976; 15(2): $157-65$.

6. Wearden AJ, Tarrier N, Barrowclough C, Zastowny TR. Rahill AA. A review of expressed emotion research in health care. Clin Psychol Rev 2000; 20(5): 633-66.
7. Mohapatra S. Expressed emotion in psychiatric disorders. Eastern J Psychiatr 2013; 16(1-2): 17-22.

8. Butler R, Berry K, Varese F, Bucci S. (2019). Are family warmth and positive remarks related to outcomes in psychosis? A systematic review. Psychol Med 2019; 49(8): 1250-65.

9. Magana AB, Goldstein JM, Karno M, Miklowitz DJ, Jenkins J. A brief method for assessing expressed emotion in relatives of psychiatric patients. Psychiatry Res 1986; 17(3): 203-12.

10. Kavanagh DJ, O'Halloran P, Manicavasagar V, Clark D, Piatkowska $\mathrm{O}$, Tennant $\mathrm{C}$, et al. The family attitude scale: reliability and validity of a new scale for measuring the emotional climate of families. Psychiatry Res 1997; 70(3): 185-95.

11. Leff J, Vaughn C. Expressed emotion in families: Its significance for mental illness. Guilford Press, New York. 1985.

12. Pallant J. SPSS survival manual: a step by step guide to data analysis using IBM SPSS (4 ${ }^{\text {th }}$ ed.). Aust NZ J Publ Health 2013; 37(1): 597-98.

13. Kaiser HF. An index of factorial simplicity. Psychometrika 1974; 39(1): 31-36.

14. Bartlett MS. A note on the multiplying factors for various chi square approximations. J R Stat Soc 1954; 16(1): 296-98.

15. Jackson DL, Gillaspy JA, Purc-Stephenson R. Reporting practices in confirmatory factor analysis: An overview and some recommendations. Psychol Methods 2009; 14(1): 6-23.

16. Fabrigar LR, Wegener DT, MacCallum RC, Strahan EJ. Evaluating the use of exploratory factor analysis in psychological research. Psychol Methods 1999; 4(3): 272-99.

17. Bentler PM. Comparative fit indexes in structural models. Psychol Bull 1990; 107(2): 238-46. 